\title{
Spatial and Temporal Characterization of Indoor Millimeter Wave Propagation at $24 \mathrm{GHz}$
}

\author{
Seok-hwan Min, ${ }^{1}$ Hayeon Kim, ${ }^{2}$ Haengseon Lee, ${ }^{2}$ Woo Jin Byun, ${ }^{3}$ Minsoo Kang, \\ Kwangseon Kim, ${ }^{3}$ Bong-su Kim, ${ }^{3}$ and Myung-sun Song ${ }^{3}$ \\ ${ }^{1}$ Department of Technology Planning, Electromagnetic Wave Technology Institute of Korea Radio Promotion Association (RAPA), \\ Seoul, Republic of Korea \\ ${ }^{2}$ Department of Electronic Engineering, Sogang University, 1 Sinsu-dong, Mapo-gu, Seoul, Republic of Korea \\ ${ }^{3}$ Radio Basic Research Section, Electronics and Telecommunications Research Institute, Daejon, Republic of Korea
}

Correspondence should be addressed to Haengseon Lee; leehs95@sogang.ac.kr

Received 20 June 2016; Revised 26 September 2016; Accepted 28 September 2016

Academic Editor: Ahmad Safaai-Jazi

Copyright (C) 2016 Seok-hwan Min et al. This is an open access article distributed under the Creative Commons Attribution License, which permits unrestricted use, distribution, and reproduction in any medium, provided the original work is properly cited.

\begin{abstract}
Indoor millimeter wave propagation at the frequency of $24 \mathrm{GHz}$ is studied by experimental methods. Measurements are performed to obtain temporal and spatial channel model using a channel sounder and rotating antennas in a corridor. The measured impulse responses are processed to obtain compact channel model following Saleh-Valenzuela's model. The responses are compared with those of $5.3 \mathrm{GHz}$ for the same test sites. Angular spread of $24 \mathrm{GHz}$ is found to be smaller than that of $5.3 \mathrm{GHz}$, while echoes of $24 \mathrm{GHz}$ are found to be longer than those of $5.3 \mathrm{GHz}$.
\end{abstract}

\section{Introduction}

With increasingly large demand for mobile internet data, unused frequency bands are being explored and frequency bands with reduced demand are reallocated to accommodate new wireless communication services. Among those frequency bands, millimeter wave frequencies have been given much interest due to the availability of large chunk of bands. For the introduction of new services such as fifth-generation (5G) services; however, the properties of electromagnetic waves at those bands should be known in various environment to simulate and estimate the performance of the new services, which necessitates accurate channel modeling [1].

In contrast to the wave propagation at SHF frequencies, the scattering directions at millimeter wave frequencies have narrow angles due to short wavelengths. The received signal strengths change rapidly with viewing angles of antennas. Obstacles with the dimensions comparable to or larger than the wavelength induce scattering along the specular or incoming directions [2]. Indoor or outdoor environments which encircle the base stations and user terminals are composed of many facets generating complicated scattering.
Although simple radio propagation models such as a free space model, a ground reflection model, and statistical models such as Hata's or Okumura's are useful, those models cannot give precise information on the millimeter wave band which varies rapidly with positions and directions of antennas [3].

In this paper, a procedure to obtain an indoor wave propagation model at millimeter wave band is presented. Measurement results are given at the frequency of $24 \mathrm{GHz}$, which are the impulse responses as a function of delay and angles. A channel sounder with chip rate of $300 \mathrm{MHz}$ and transmitting/receiving RF front ends is built and used to obtain impulse responses [4]. The measured data of $24 \mathrm{GHz}$ band are compared with that of $5.3 \mathrm{GHz}$. Modified SalehValenzuela's model [5] parameters are extracted which catch important features of impulse response by exponential functions. The obtained channel model enables measurement data to be processed such that receiving voltages can be retrieved as a function of time and angle. The measurement setup is given in Section 2, measured data is given in Section 3 , and modified S-V model parameters are extracted in Section 4 . 


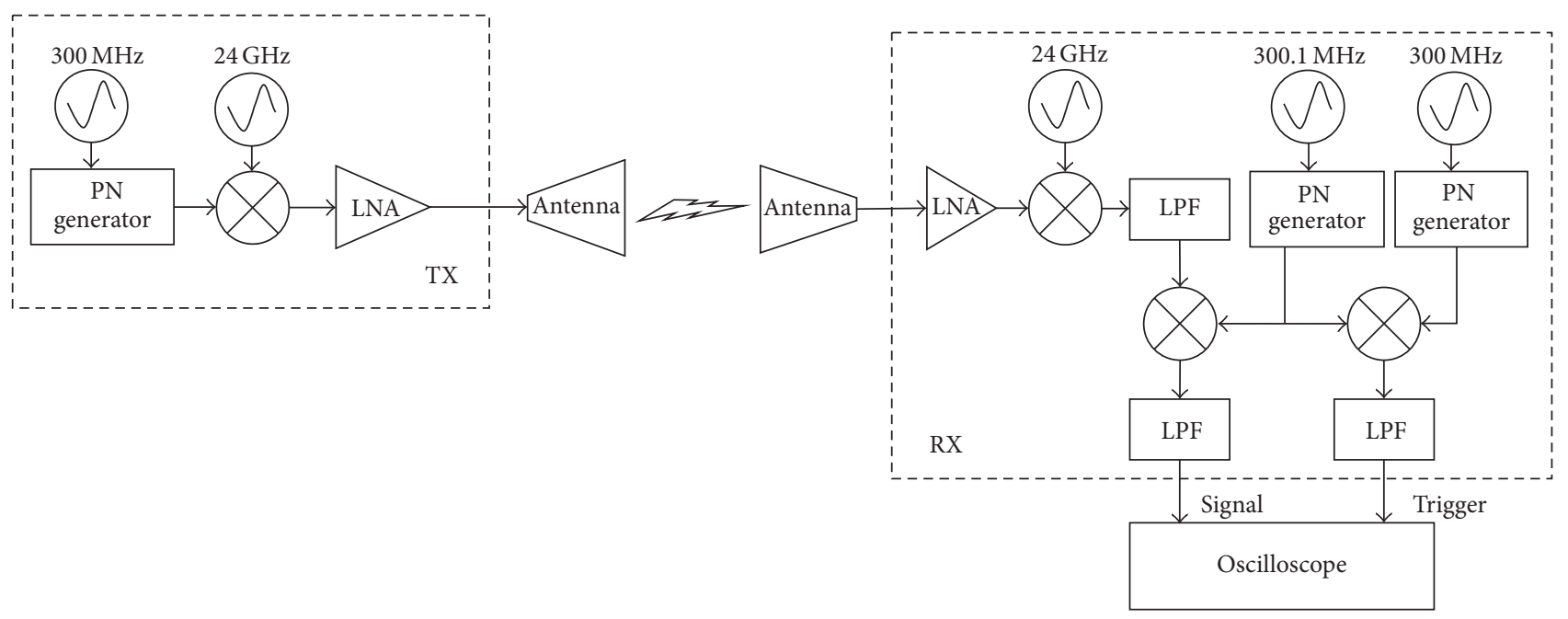

FIGURE 1: A schematic diagram of a sliding correlator used in the channel sounding. The oscillators are synchronized by an external $10 \mathrm{MHz}$ reference clock which is not shown in the figure.

\section{Measurement Setup}

To obtain the impulse response of $24 \mathrm{GHz}$, a sliding correlator channel sounder is built which generates 9-bit maximal pseudorandom binary sequence as in Figure 1. The chip rate of the transmitter is $300 \mathrm{MHz}$ and that of the receiver is $300.1 \mathrm{MHz}$. The pseudorandom noise (PN) generator on the transmitter spreads the $24 \mathrm{GHz}$ continuous wave signals into wideband ones, and those on the receiver despread them. The difference of the chip rates makes the cross-correlated output on the receiver show channel impulse responses with elongated time scale, whose period is the inverse of the difference frequency. The number of bits is chosen to maximize signal-to-noise ratio on the receiver side. The transmitter and the receiver are synchronized with the external reference signal of $10 \mathrm{MHz}$ which is not shown in the figure. A $300 \mathrm{MHz} \mathrm{PN}$ generator of the receiver synchronized with that of the transmitter and acts as trigger signals which indicate the start of impulse responses. The PLLs attached to the oscillators $300 \mathrm{MHz}, 300.1 \mathrm{MHz}$, and $24 \mathrm{GHz}$ are all fed by the same $10 \mathrm{MHz}$ reference clock. A programmable logic device is used to facilitate the change of specifications.

The RF front ends of the channel sounders are configured to measure $24 \mathrm{GHz}$ and $5.3 \mathrm{GHz}$ impulse responses at many positions in the indoor environment as in Figure 2. Impulse responses are recorded as the receiver is moved from the nearby position of the transmitter to far places. Table 1 shows coordinates of positions of the transmitter and the receiver. The height of ceiling of the corridor from the floor is $2.57 \mathrm{~m}$.

Figure 3 shows the photos of the test site, which is a corridor $60 \mathrm{~m}$ long and $2.5 \mathrm{~m}$ wide. Impulse responses are obtained with the positions of the receiver changed. The amplitude variations of the received signals along the receiver positions are measured separately by a spectrum analyzer due to the narrow dynamic range of the oscilloscope. To extract spatial and temporal responses, impulse responses are recorded for 36 arrival angles by rotating the receiving antenna by 10 degrees after each measurement at a given position. The angular measurements are performed at 13 positions as shown in Figure 3. The transmit power is $10 \mathrm{dBm}$, and total cable losses for the transmitter and the receiver are $10 \mathrm{~dB}$.

The antennas used for $24 \mathrm{GHz}$ are standard gain horns with $21 \mathrm{dBi}$ gain and waveguide size WR42 as shown in Figure 4. The radiation pattern for the antennas is also shown. For the frequency of $5.3 \mathrm{GHz}$, antennas are replaced with patch arrays with the same peak gain whose radiation pattern is also shown.

\section{Measured Results}

The measured impulse responses are given in Figure 5 as a function of arrival angles and time delays. The time axis is adjusted to take into account the time dilation of the sliding correlator which is due to the chip rate difference between the PN generator of the transmitter and that of the receiver. The output time scale of the sliding correlator is elongated by the ratio of the chip rate to the difference of the chip rates. The colors show relative strengths of the received signals normalized by the maximum value at each receiver's position for the range of $50 \mathrm{~dB}$. Impulse responses at the frequencies of $24 \mathrm{GHz}$ and $5.3 \mathrm{GHz}$ are shown side by side to compare them. The reference direction for angles of arrival is defined in Figure 5(a). Although the center frequencies are different, the time delay of peak received signals is similar.

The measured impulse responses are compared with one another. They are sorts of bistatic radar images in that the time axis is proportional to the distances to obstacles in the corridor as shown in Figure 6 which is the impulse response 


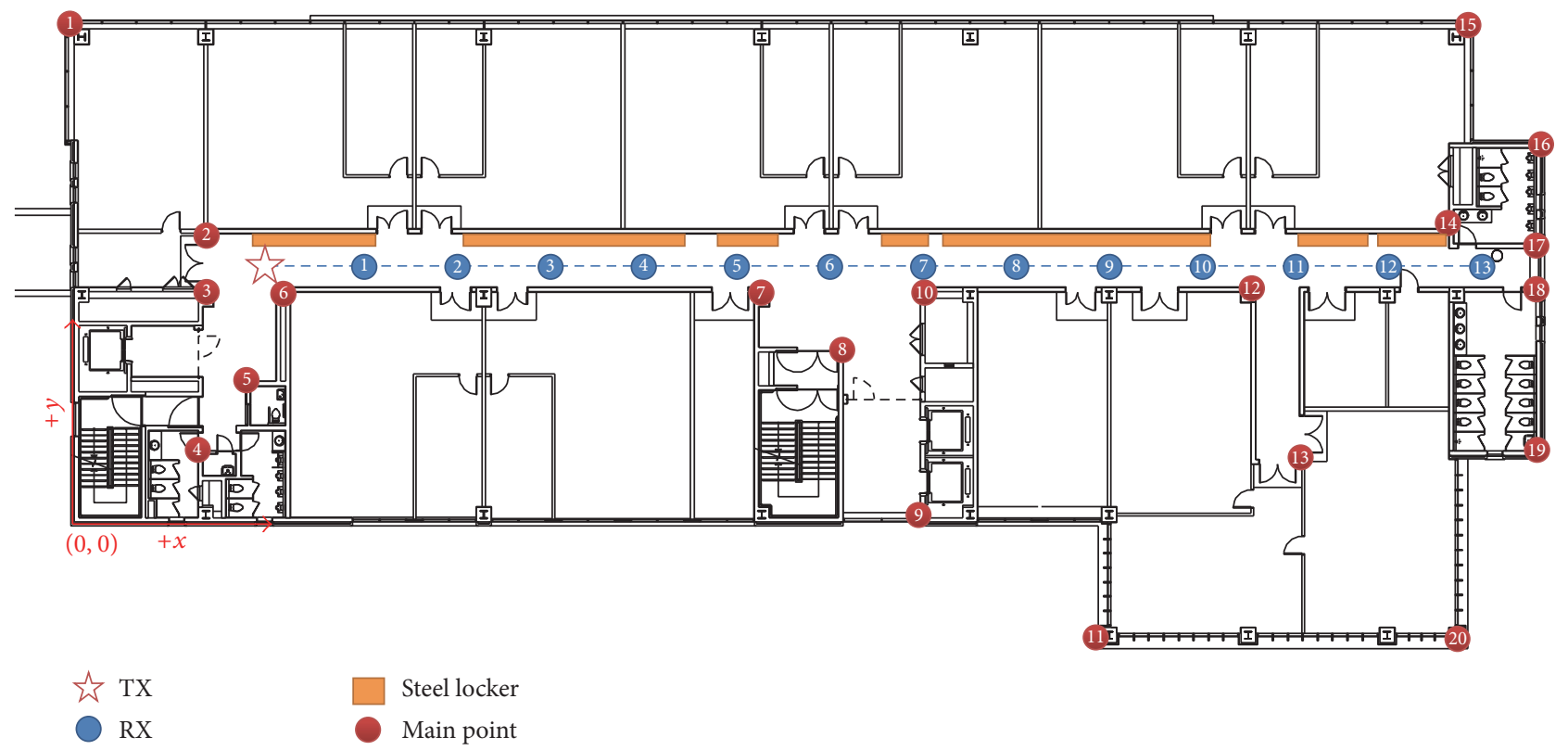

Figure 2: Map of the test site. The origin of the coordinate is at the lower left.

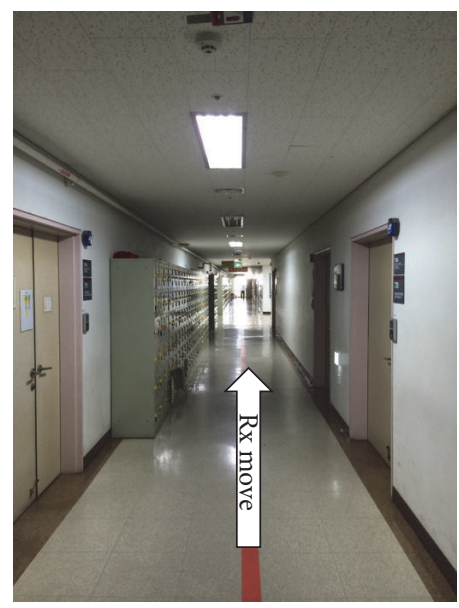

(a) The test site

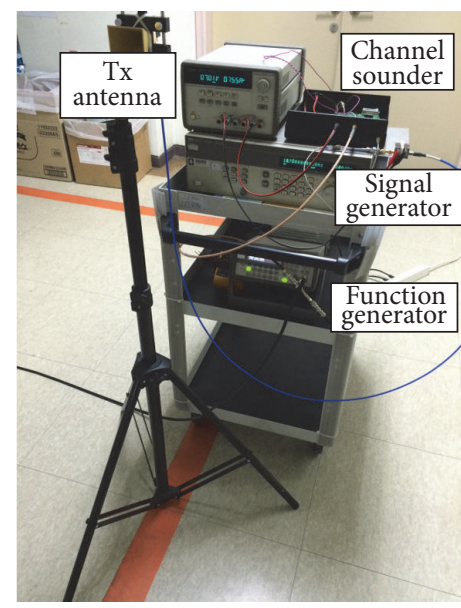

(b) Transmitter

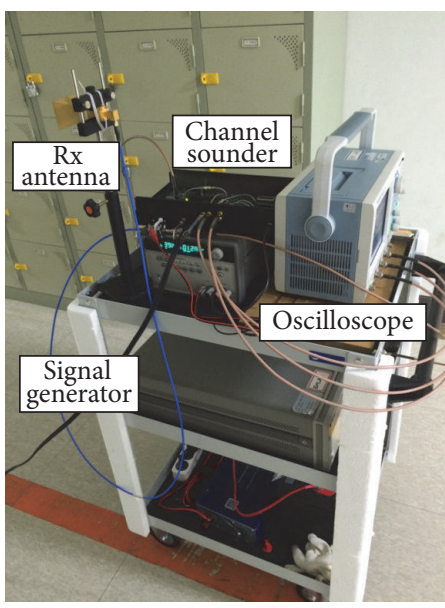

(c) Receiver

FIGURE 3: The test site and the measurement setup.

at the position of RX1. During the first-round trip time for the transmitted signals $(0 \sim 0.42 \mu \mathrm{s})$, the impulse response at the angle of 180 degree shows time history of scattered waves from the obstacles along the corridor. As the corridor is relatively long with narrow widths, it can be considered as a one-dimensional scattering problem. Large reflections or scatterings are observed from the walls at either end of the corridor, side walls of lockers, and steel doors of the rooms. The attenuation of the signal level through the wall is measured to be about $50 \mathrm{~dB}$, which means that the scattering in the corridor can be assumed to be nearly one-dimensional scattering. The peak positions in the impulse responses can be identified with the scattering point in the corridor as shown in Figure 6(b), which helps to form impulse responses at other positions.

Figure 7 shows the variation of the signal strength as the receiver is moved from the position near the transmitter to the right end of the corridor. One received signal can be retrieved by the product of the signal strength and impulse responses in Figure 5.

\section{Extracted Channel Model Parameters}

Although the measurement data are important enough, their sizes are cumbersome to utilize in simulations for wireless 


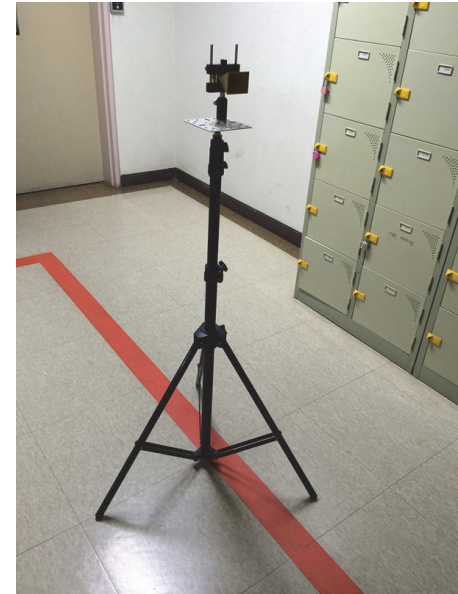

(a) Standard gain horn for $24 \mathrm{GHz}$

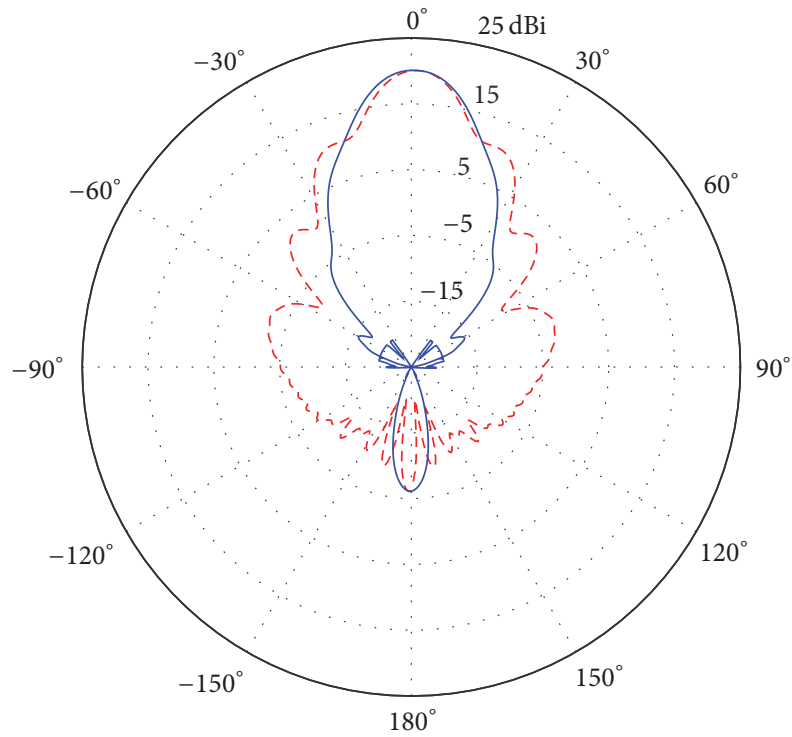

- - E-plane - H-plane

(c) $24 \mathrm{GHz}$ horn

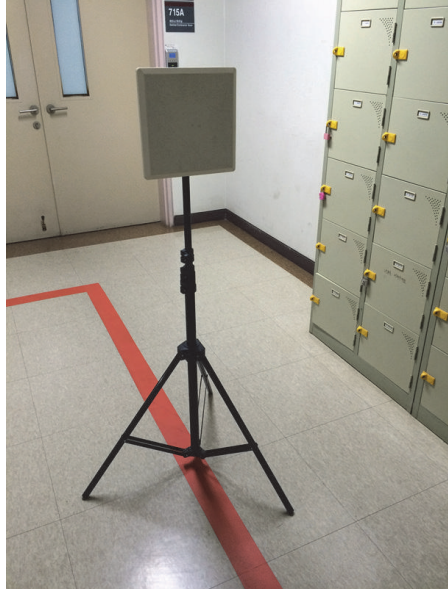

(b) Patch array antenna for $5.3 \mathrm{GHz}$

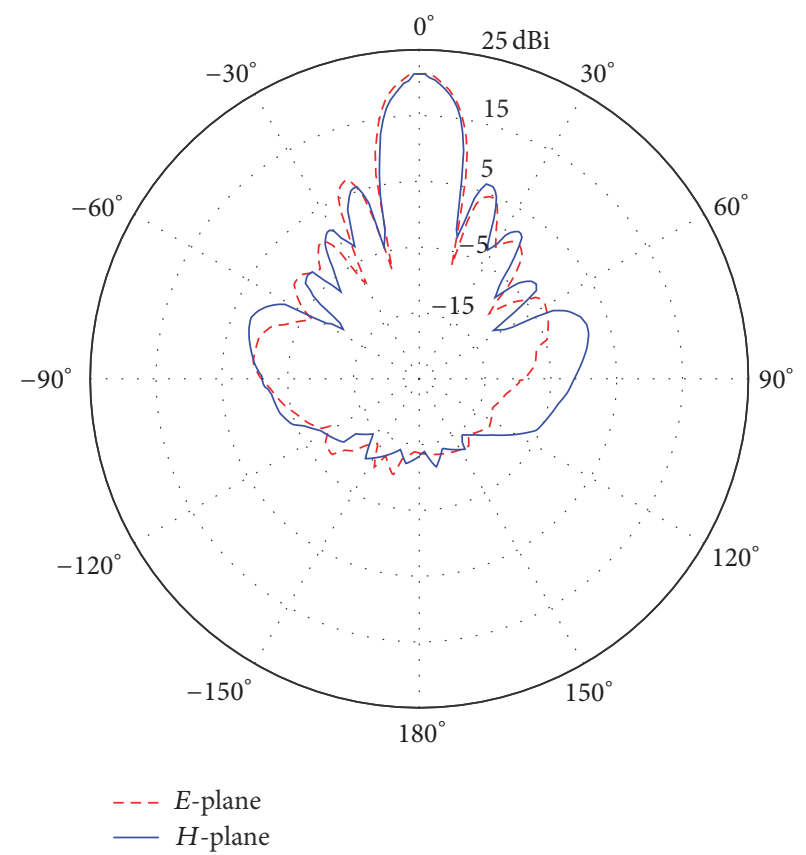

(d) $5.3 \mathrm{GHz}$ patch array antenna

FIGURE 4: Antennas and radiation patterns of the channel sounder.

communications. To alleviate the computational burden, curve fitting is used like Saleh-Valenzuela's channel model $[5,6]$ to simplify the impulse responses which are composed of clusters of delta functions. Clusters of delta functions are approximated collectively by decaying exponential functions. Besides, the angular dependence of the impulse responses is also taken into account by modifying $\mathrm{S}-\mathrm{V}$ model. The modified S-V model at one receiver position is as follows:

$$
h(t, \theta)=\sum_{i} A_{i} F_{i}(t) G_{i}(\theta),
$$

where

$$
\begin{gathered}
F_{i}(t)= \begin{cases}\exp \left(\frac{t-\tau_{i}}{\tau_{L, i}}\right), & \text { for } t<\tau_{i}, \\
\exp \left(-\frac{t-\tau_{i}}{\tau_{R, i}}\right), & \text { for } t>\tau_{i},\end{cases} \\
G_{i}(t)= \begin{cases}\exp \left(\frac{\theta-\theta_{i}}{\theta_{L, i}}\right), & \text { for } \theta<\theta_{i}, \\
\exp \left(-\frac{\theta-\theta_{i}}{\theta_{R, i}}\right), & \text { for } \theta>\theta_{i} .\end{cases}
\end{gathered}
$$




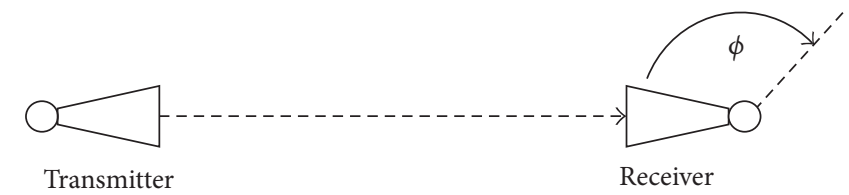

(a) Definition of angle of arrivals

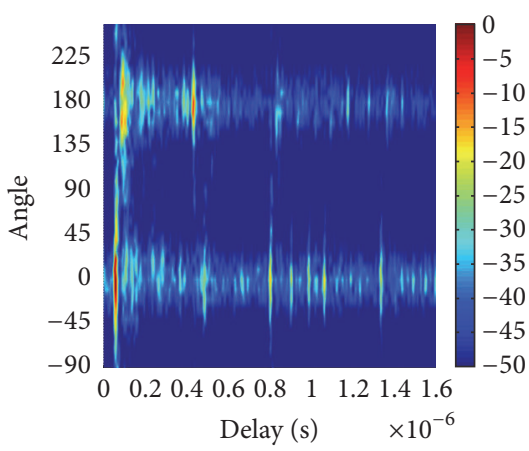

(b) Impulse response of $24 \mathrm{GHz}$ at $\mathrm{RX1}$

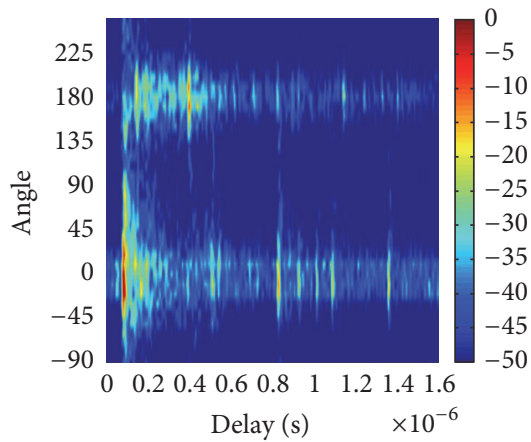

(d) Impulse response of $24 \mathrm{GHz}$ at $\mathrm{RX} 3$

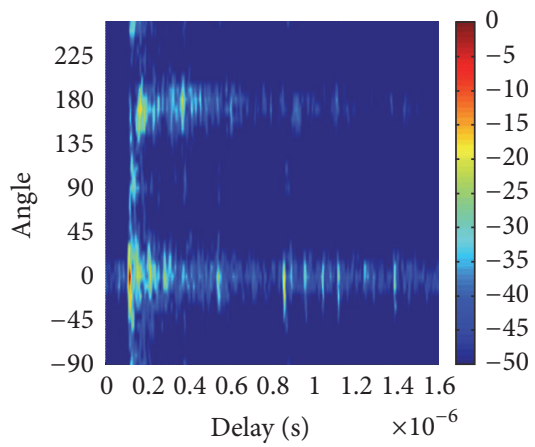

(f) Impulse response of $24 \mathrm{GHz}$ at RX5

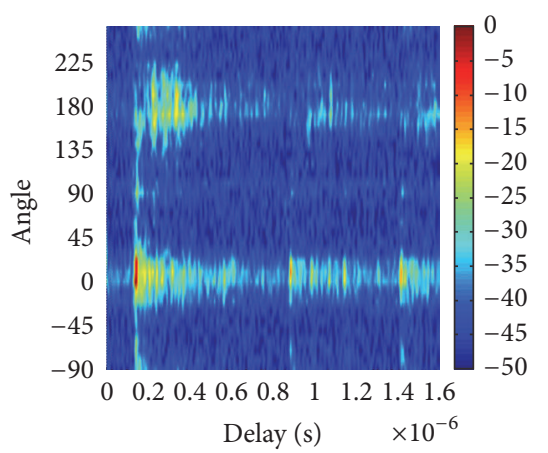

(h) Impulse response of $24 \mathrm{GHz}$ at RX7

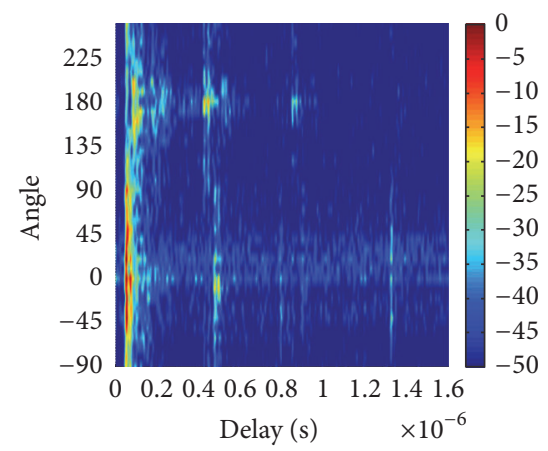

(c) Impulse response of $5.3 \mathrm{GHz}$ at $\mathrm{RX} 1$

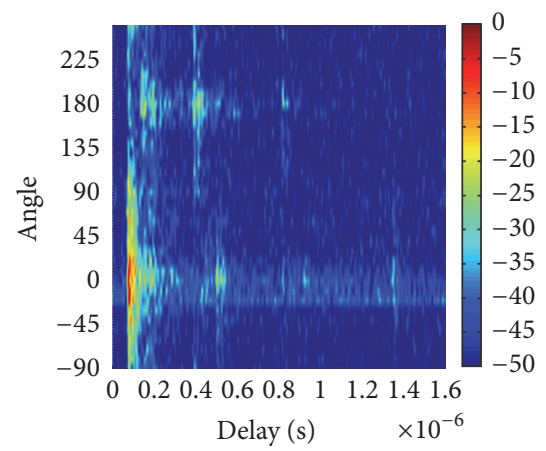

(e) Impulse response of $5.3 \mathrm{GHz}$ at RX3

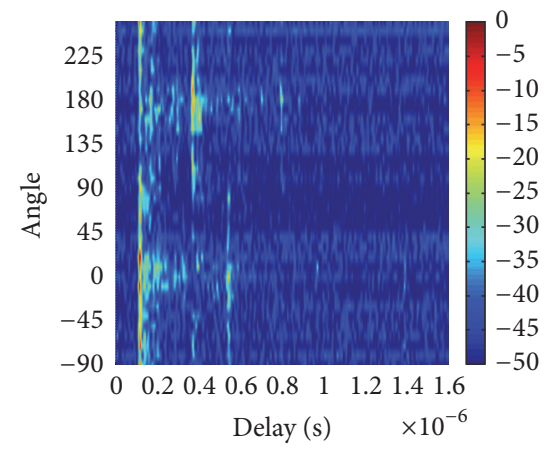

(g) Impulse response of $5.3 \mathrm{GHz}$ at RX5

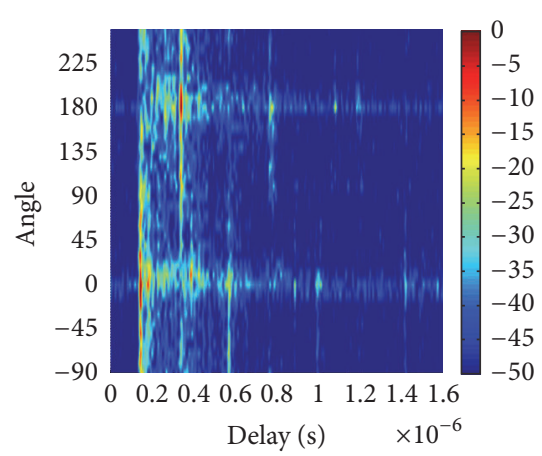

(i) Impulse response of $5.3 \mathrm{GHz}$ at $\mathrm{RX7}$

FIgure 5: Continued. 


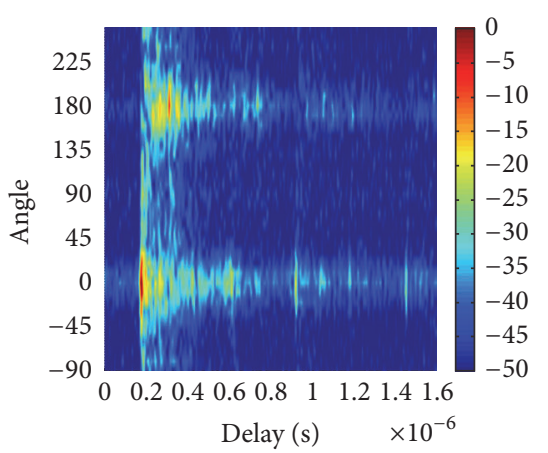

(j) Impulse response of $24 \mathrm{GHz}$ at $\mathrm{RX} 9$

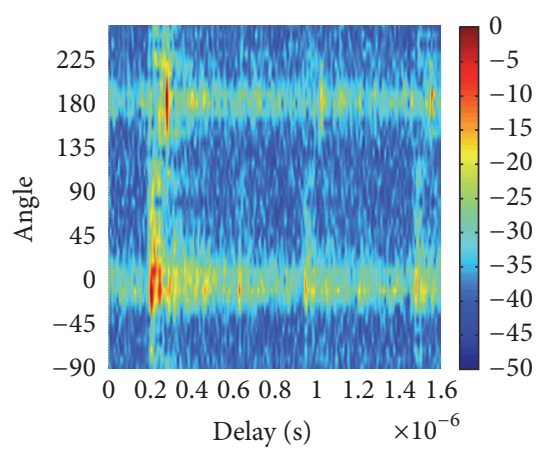

(1) Impulse response of $24 \mathrm{GHz}$ at $\mathrm{RX11}$

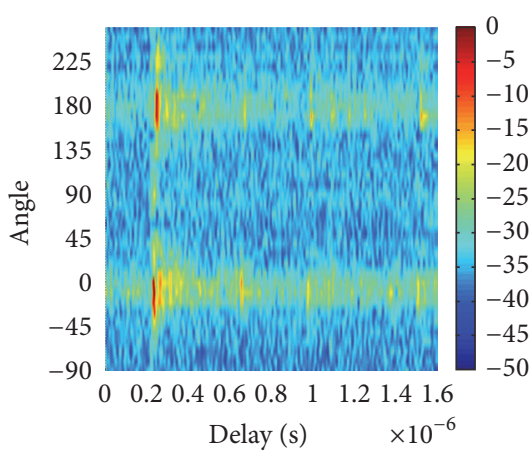

(n) Impulse response of $24 \mathrm{GHz}$ at $\mathrm{RX13}$

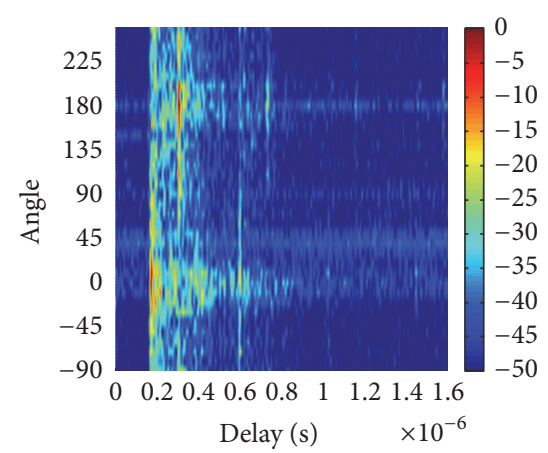

(k) Impulse response of $5.3 \mathrm{GHz}$ at $\mathrm{RX} 9$

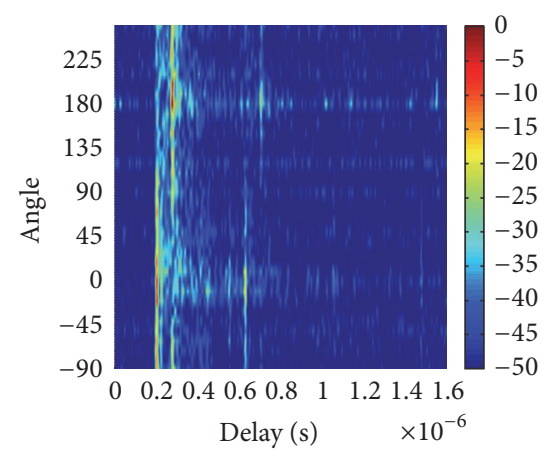

(m) Impulse response of $5.3 \mathrm{GHz}$ at RX11

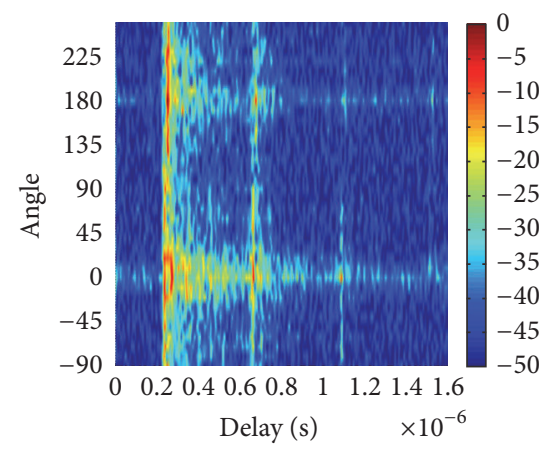

(o) Impulse response of $5.3 \mathrm{GHz}$ at $\mathrm{RX13}$

Figure 5: Measurement results of $24 \mathrm{GHz}$ signals at 13 receiver positions.

The model parameter $\tau_{i}$ is $i$ th peak delay times, $\tau_{L, i}$ is the slope parameter prior to $\tau_{i}$, and $\tau_{R, i}$ is the slope parameter after that time. Likewise, the model parameter $\theta_{i}$ is $i$ th azimuthal angle in which direction a peak power is observed. The coefficients $A_{i}$ represent relative strengths of the peak powers. The procedure to extract model parameters is summarized by the flow graph in Figure 8 .

Following the procedure, channel parameters for the position of RX1 are extracted as shown in Figure 9 with the dynamic range of $25 \mathrm{~dB}$. The responses of $24 \mathrm{GHz}$ signals have longer echoes than those of $5.3 \mathrm{GHz}$ in time in that higher order reflections with considerable strength can be observed. Although the beam widths of the $24 \mathrm{GHz}$ and $5.3 \mathrm{GHz}$ antennas are similar, the angular spread of the $5.3 \mathrm{GHz}$ impulse response is larger than that of $24 \mathrm{GHz}$.
Table 2 summarizes channel parameters extracted at the position of RX1. The amplitudes A's are normalized by peak value at each receiver position. As shown in Figure 9, the delay parameters $\tau_{i}$ 's of $24 \mathrm{GHz}$ are more widely spread than those of $5.3 \mathrm{GHz}$, whereas the spread of angular parameters of $5.3 \mathrm{GHz}$ is larger than that of $24 \mathrm{GHz}$.

\section{Conclusion}

In this paper, angular and temporal characterization of $24 \mathrm{GHz}$ millimeter wave propagation is made experimentally. Comparison with $5.3 \mathrm{GHz}$ case is also made to emphasize the properties of millimeter wave signals. To obtain impulse response as a function of time and angle, a channel sounder with rotating antenna is used. The measured data show that 

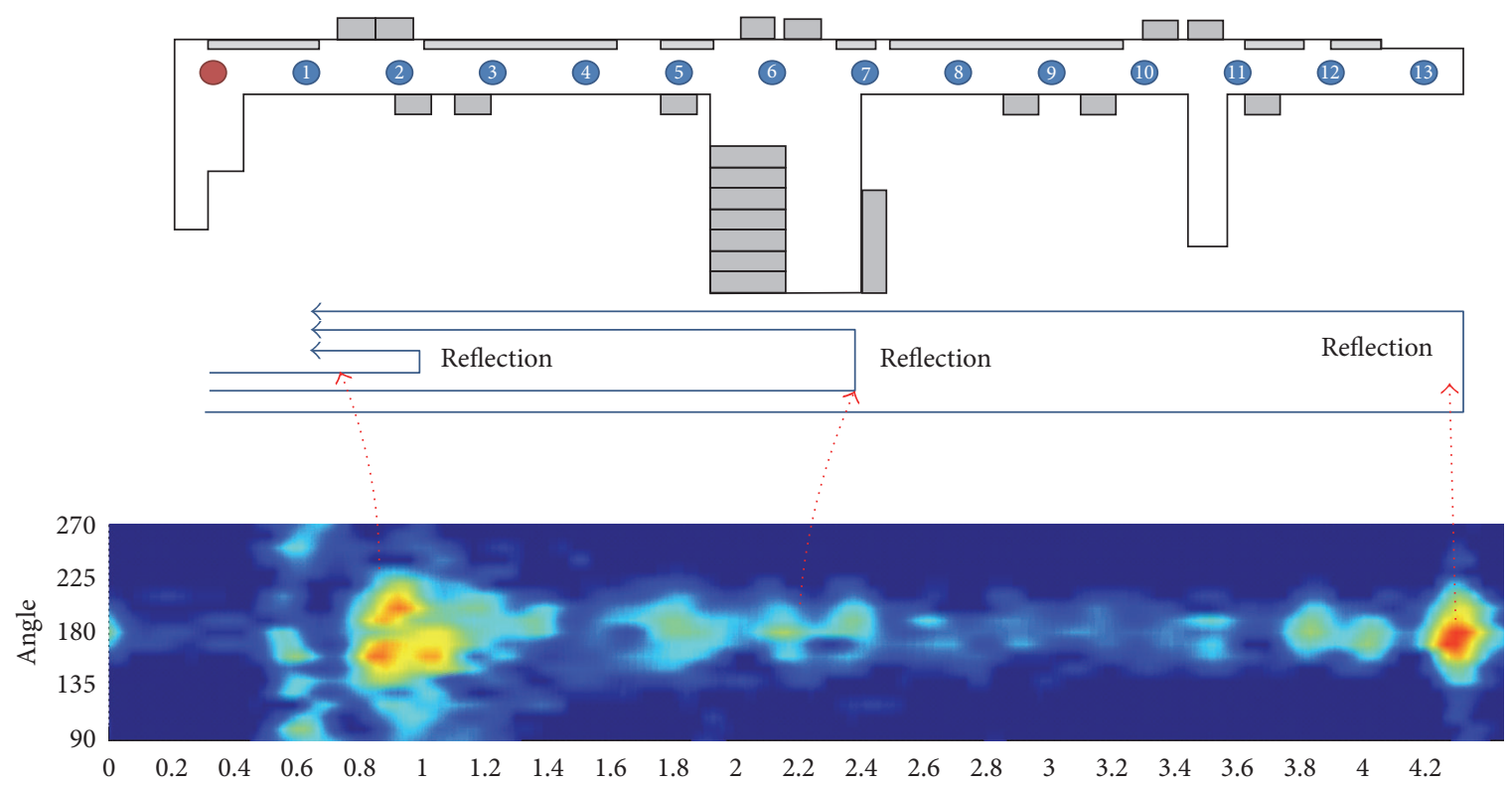

(a) Close-up of the impulse response with the receiver pointed to the right end of the corridor

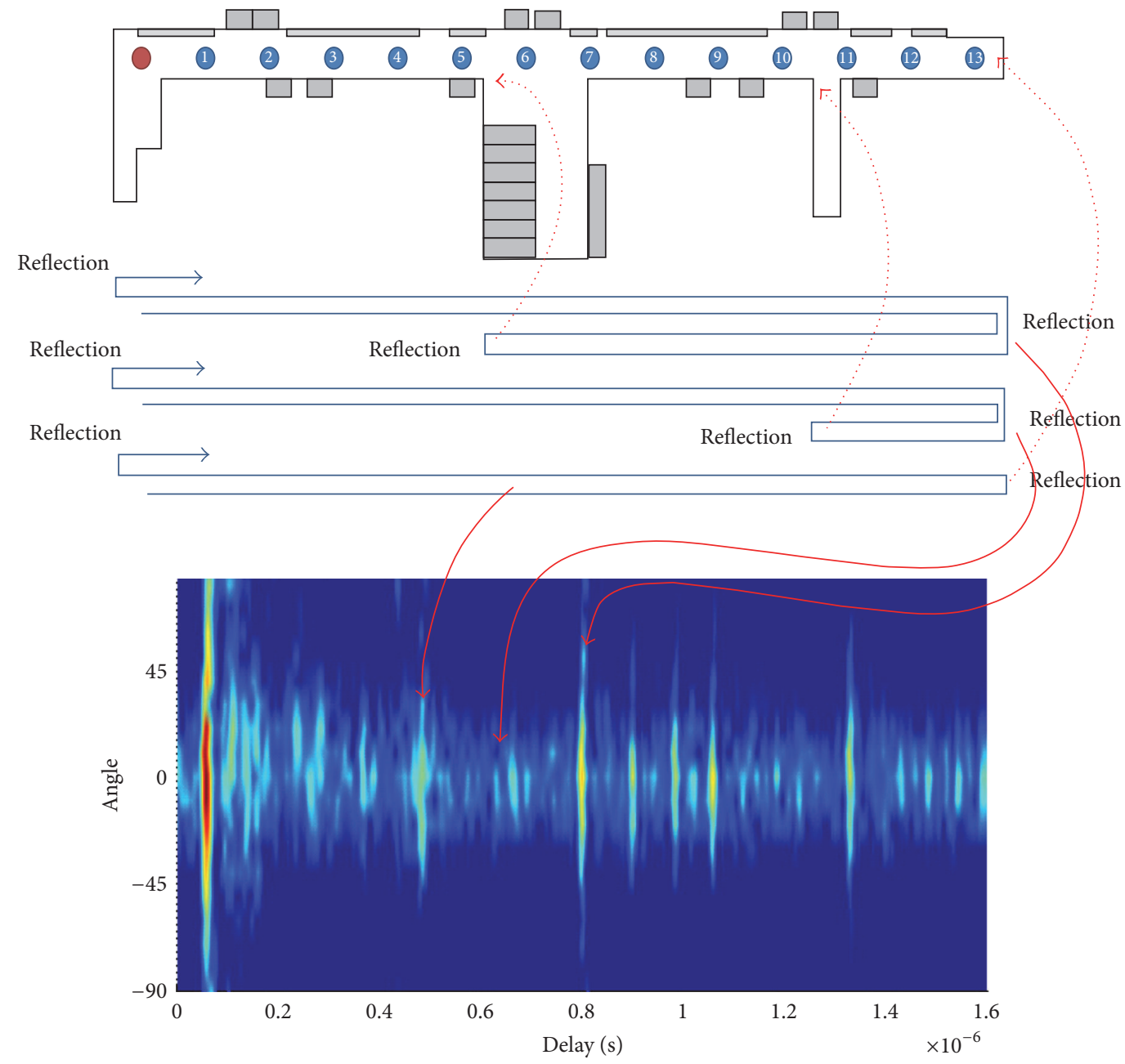

(b) The impulse response with the receiver pointed to the transmitter

FIGURE 6: Impulse response obtained at RX1. 
TABLE 1: Coordinates of the transmitter, the receiver, and corners of the test site. Metric unit is used.

(a) Coordinates of the transmitter and the receiver

\begin{tabular}{lcc}
\hline Site & $x$ & $y$ \\
\hline $\mathrm{Tx}$ & 9.49 & 12.39 \\
$\mathrm{Rx} 1$ & 14 & 12.39 \\
$\mathrm{R} x 2$ & 18.5 & 12.39 \\
$\mathrm{Rx} 3$ & 23 & 12.39 \\
$\mathrm{R} x 4$ & 27.5 & 12.39 \\
$\mathrm{R} x 5$ & 32 & 12.39 \\
$\mathrm{R} x 6$ & 36.5 & 12.39 \\
$\mathrm{R} x 7$ & 41 & 12.39 \\
$\mathrm{R} x 8$ & 45.5 & 12.39 \\
$\mathrm{R} x 9$ & 50 & 12.39 \\
$\mathrm{R} x 10$ & 54.5 & 12.39 \\
$\mathrm{R} x 11$ & 59 & 12.39 \\
$\mathrm{R} x 12$ & 63.5 & 12.39 \\
$\mathrm{Rx} 13$ & 68 & 12.39 \\
\hline
\end{tabular}

(b) Coordinates of the corners of the test site

\begin{tabular}{|c|c|c|}
\hline \# & $x$ & $y$ \\
\hline 1 & 0 & 24 \\
\hline 2 & 6.79 & 13.81 \\
\hline 3 & 6.79 & 11.3 \\
\hline 4 & 6.79 & 4.74 \\
\hline 5 & 8.57 & 6.85 \\
\hline 6 & 10.02 & 11.3 \\
\hline 7 & 33.45 & 11.3 \\
\hline 8 & 37 & 8.45 \\
\hline 9 & 40.57 & 0.34 \\
\hline 10 & 40.57 & 11.3 \\
\hline 11 & 49.06 & -5.8 \\
\hline 12 & 56.56 & 11.34 \\
\hline 13 & 58.55 & 3.18 \\
\hline 14 & 65.69 & 13.81 \\
\hline 15 & 67 & 24 \\
\hline 16 & 70 & 18 \\
\hline 17 & 70 & 13 \\
\hline 18 & 70 & 11.3 \\
\hline 19 & 70 & 3.32 \\
\hline 20 & 66.5 & -5.8 \\
\hline
\end{tabular}



FIGURE 7: Received power levels of $24 \mathrm{GHz}$ and $5.3 \mathrm{GHz}$ signals with the receiver moved from the transmitter to the right end of the corridor.

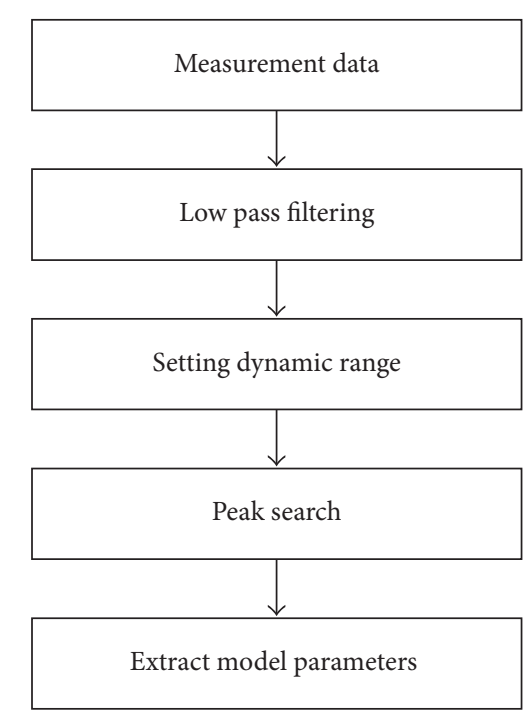

FIGURE 8: Procedures to extract model parameters.

angular spread of $24 \mathrm{GHz}$ signal is smaller than that of $5.3 \mathrm{GHz}$, while echoes of $24 \mathrm{GHz}$ are longer than those of $5.3 \mathrm{GHz}$. A procedure to extract modified S-V model parameters is also presented to facilitate simulation of millimeter wave communication systems.

\section{Competing Interests}

The authors declare that there is no conflict of interests regarding the publication of this article.

\section{Acknowledgments}

This work was supported by the ICT R\&D program of MSIP/IITP [B0101-16-0222, Development of Core Technologies to Improve Spectral Efficiency for Mobile Big-Bang]. 
TABLE 2: Channel parameters extracted at the position of RX1.

(a) Channel parameters for $24 \mathrm{GHz}$

\begin{tabular}{lccccccc}
\hline Cluster & $A[\mathrm{~dB}]$ & $\tau_{i}[\mathrm{~ns}]$ & $\tau_{L, i}[\mathrm{~ns}]$ & $\tau_{R, i}[\mathrm{~ns}]$ & $\theta_{i}[\mathrm{deg}]$ & $\theta_{L, i}[\mathrm{deg}]$ & $\theta_{R, i}[\mathrm{deg}]$ \\
\hline 1 & -0.21 & 57 & 17.0 & 13.0 & 0 & 63.98 & 64.69 \\
2 & -18.83 & 104 & 32.4 & 70.5 & 180 & 86.45 & 52 \\
3 & -28.73 & 136 & 11.2 & 30.7 & 180 & 30.02 & 48.89 \\
4 & -26.74 & 182 & 80.2 & 20.1 & 180 & 21.27 & 42.94 \\
5 & -23.87 & 215 & 25.3 & 18.9 & 180 & 31.08 & 35.96 \\
6 & -28.4 & 235 & 21.0 & 51.0 & 180 & 54.18 & 33.74 \\
7 & -24.91 & 383 & 21.6 & 14.6 & 180 & 33.58 & 40.1 \\
8 & -26.97 & 403 & 13.7 & 24.1 & 180 & 42.77 & 41.51 \\
9 & -8.8 & 430 & 20.5 & 16.9 & 180 & 52.07 & 46.01 \\
10 & -16.05 & 798 & 11.8 & 11.8 & 0 & 62.69 & 46.58 \\
11 & -17 & 1060 & 8.7 & 11.5 & 0 & 22.57 & 42.25 \\
\hline
\end{tabular}

(b) Channel parameters for $5.3 \mathrm{GHz}$

\begin{tabular}{lccccccc}
\hline Cluster & $A[\mathrm{~dB}]$ & $\tau_{i}[\mathrm{~ns}]$ & $\tau_{L, i}[\mathrm{~ns}]$ & $\tau_{R, i}[\mathrm{~ns}]$ & $\theta_{i}[\mathrm{deg}]$ & $\theta_{L, i}[\mathrm{deg}]$ & $\theta_{R, i}[\mathrm{deg}]$ \\
\hline 1 & -0.22 & 62 & 18.5 & 9.7 & 0 & 119.77 & 112.29 \\
2 & -6.56 & 81 & 19.7 & 26.2 & 0 & 65.32 & 133.85 \\
3 & -16.09 & 101 & 18.8 & 39.1 & 0 & 38.64 & 50.85 \\
4 & -15.8 & 147 & 19.6 & 12.5 & 0 & 53.84 & 62.21 \\
5 & -30.6 & 156 & 31.0 & 11.3 & 180 & 149.23 & 35.09 \\
6 & -27.37 & 173 & 18.6 & 20.1 & 180 & 64 & 41.22 \\
7 & -28.6 & 193 & 44.0 & 23.3 & 180 & 78.57 & 64.48 \\
8 & -17.41 & 404 & 12.8 & 11.2 & 180 & 41.39 & 79.41 \\
9 & -12.5 & 426 & 16.1 & 12.4 & 180 & 37.63 & 44.78 \\
10 & -28.64 & 444 & 19.6 & 22.3 & 180 & 28.06 & 117.97 \\
11 & -15.85 & 488 & 10.2 & 8.3 & 0 & 73.99 & 74.64 \\
12 & -29.71 & 491 & 28.0 & 13.1 & 180 & 94.47 & 88.78 \\
13 & -19.25 & 508 & 7.2 & 10.7 & 0 & 69.82 & 56.56 \\
14 & -19.89 & 832 & 11.5 & 9.0 & 180 & 54.69 & 55.87 \\
\hline
\end{tabular}




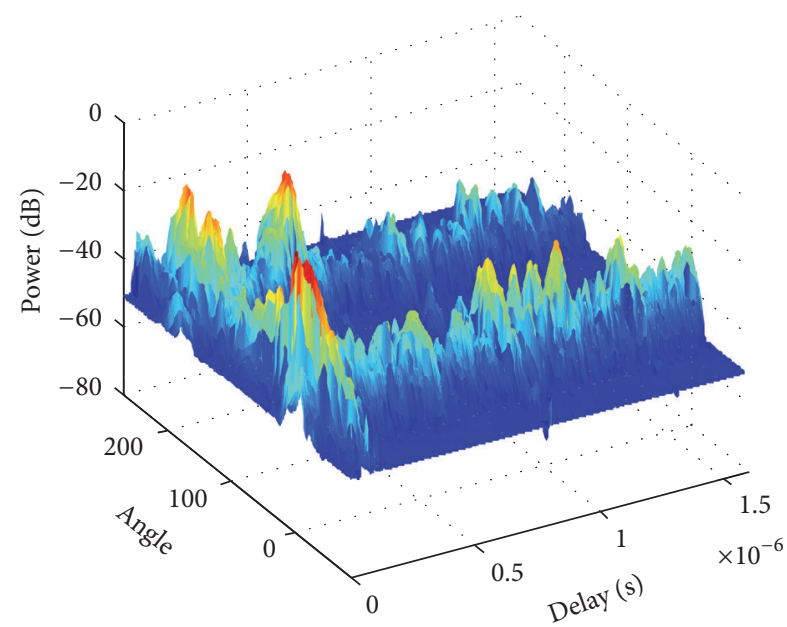

(a) Measurement results of $24 \mathrm{GHz}$

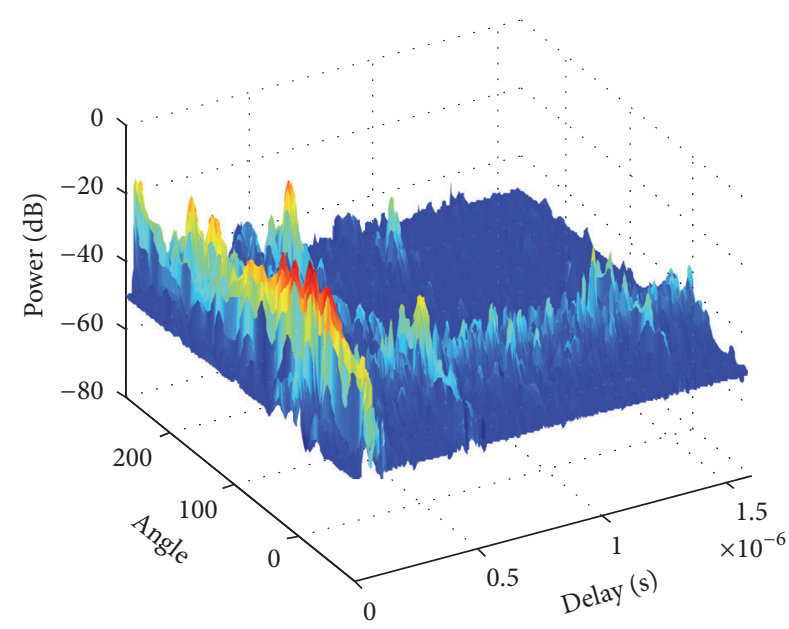

(c) Measurement results of $5.3 \mathrm{GHz}$

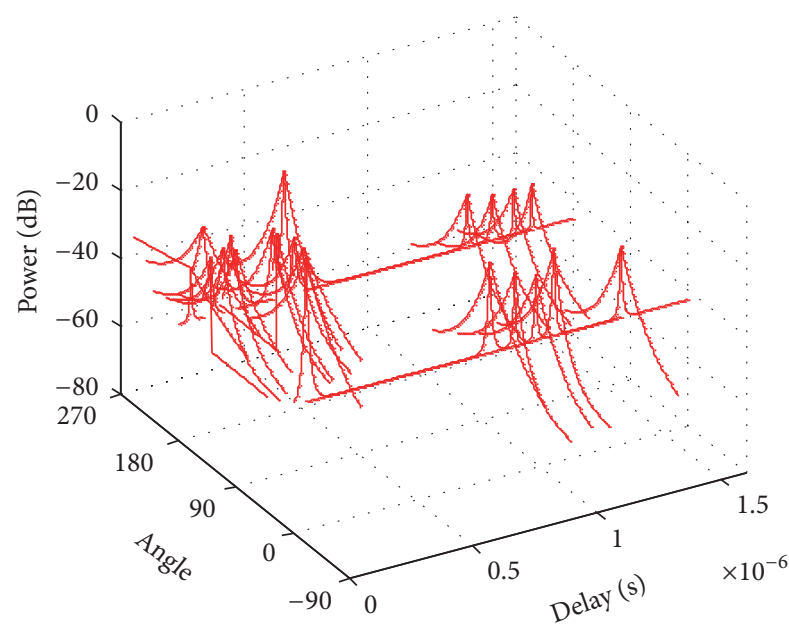

(b) Extracted S-V model functions for $24 \mathrm{GHz}$

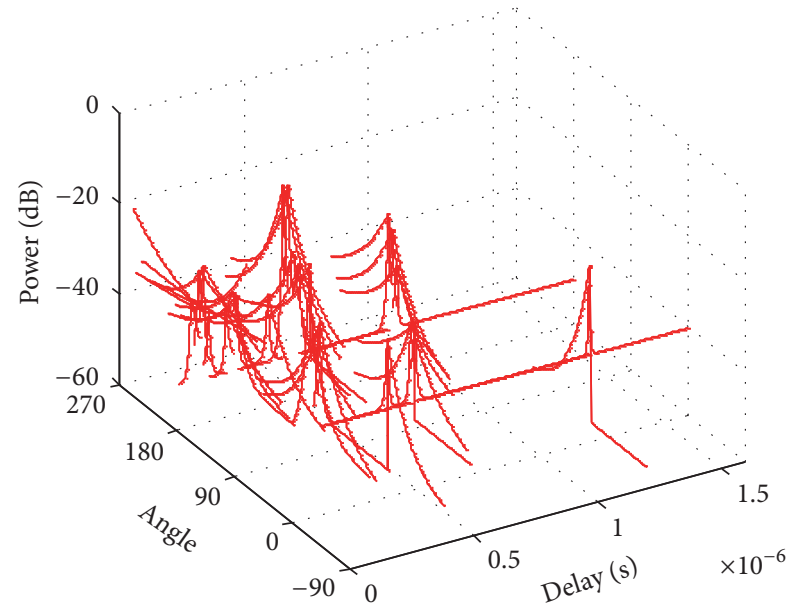

(d) Extracted S-V model functions for $5.3 \mathrm{GHz}$

FIGURE 9: Measurements and extracted channel model parameters.

\section{References}

[1] M. R. Akdeniz, Y. Liu, M. K. Samimi et al., "Millimeter wave channel modeling and cellular capacity evaluation," IEEE Journal on Selected Areas in Communications, vol. 32, no. 6, pp. 1164-1179, 2014.

[2] K. E. Torrance and E. M. Sparrow, "Theory for off-specular reflection from roughened surfaces," Journal of the Optical Society of America, vol. 57, no. 9, pp. 1105-1114, 1967.

[3] H. Xu, V. Kukshya, and T. S. Rappaport, "Spatial and temporal characteristics of 60-GHZ indoor channels," IEEE Journal on Selected Areas in Communications, vol. 20, no. 3, pp. 620-630, 2002.

[4] R. J. Pirkl and G. D. Durgin, "Optimal sliding correlator channel sounder design," IEEE Transactions on Wireless Communications, vol. 7, no. 9, pp. 3488-3497, 2008.

[5] A. A. M. Saleh and R. A. Valenzuela, "A statistical model for indoor multipath propagation," IEEE Journal on Selected Areas in Communications, vol. 5, no. 2, pp. 128-137, 1987.
[6] Q. H. Spencer, B. D. Jeffs, M. A. Jensen, and A. L. Swindlehurst, "Modeling the statistical time and angle of arrival characteristics of an indoor multipath channel," IEEE Journal on Selected Areas in Communications, vol. 18, no. 3, pp. 347-360, 2000. 


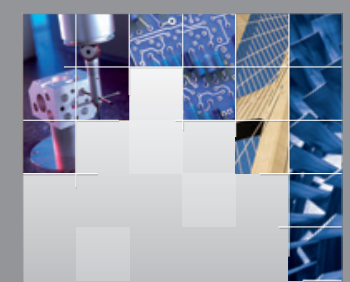

\section{Enfincering}
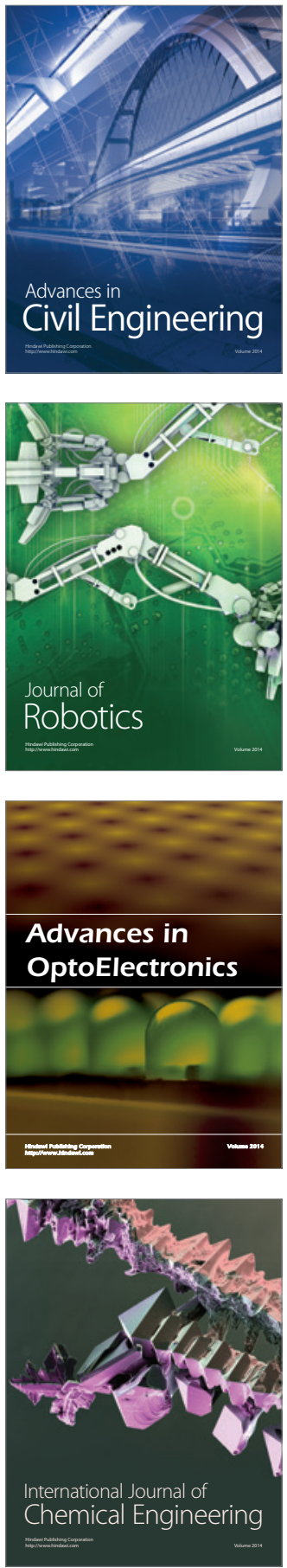

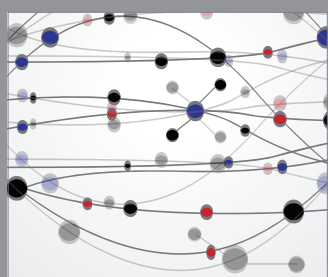

The Scientific World Journal

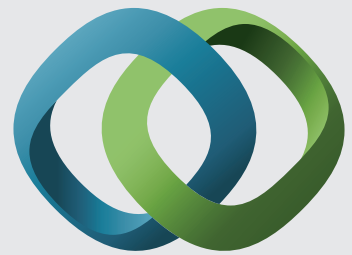

\section{Hindawi}

Submit your manuscripts at

http://www.hindawi.com
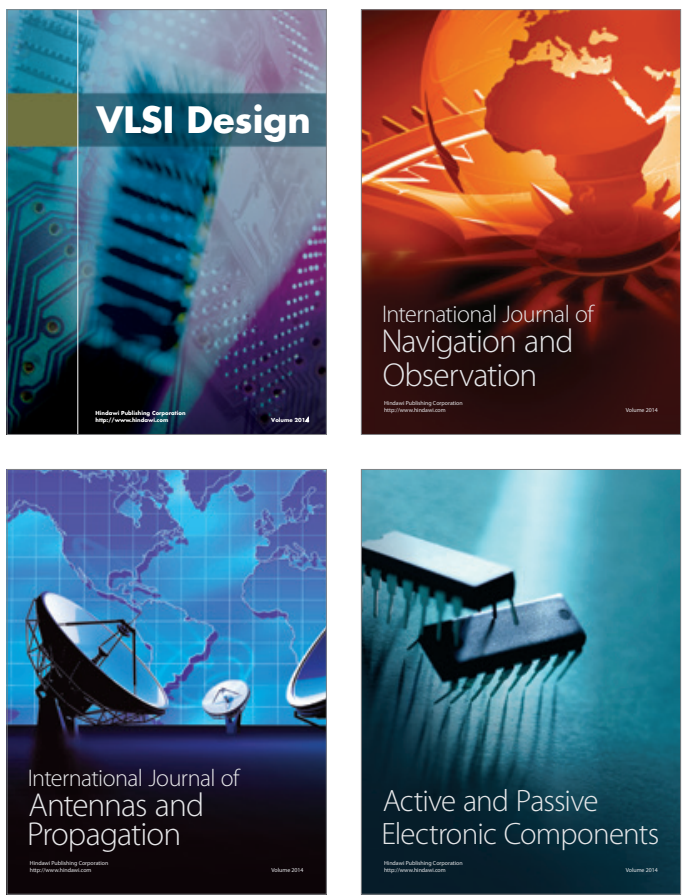
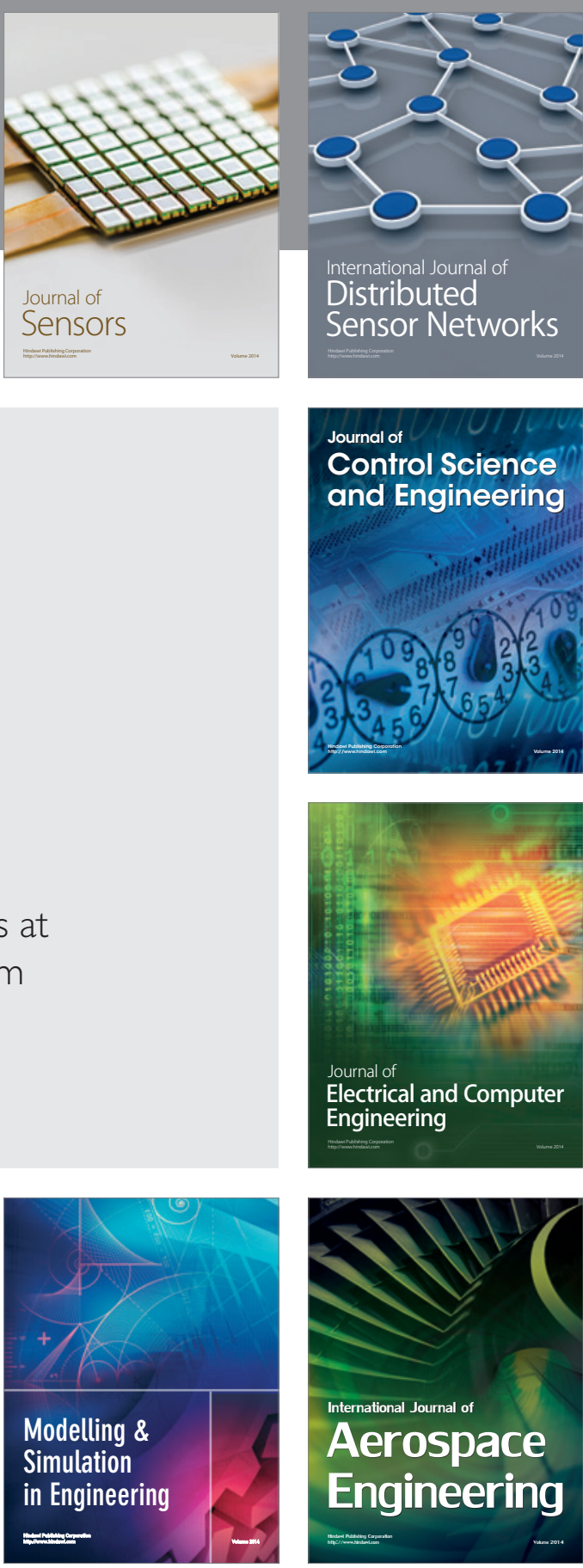

International Journal of

Distributed

Sensor Networks

Journal of

Control Science

and Engineering
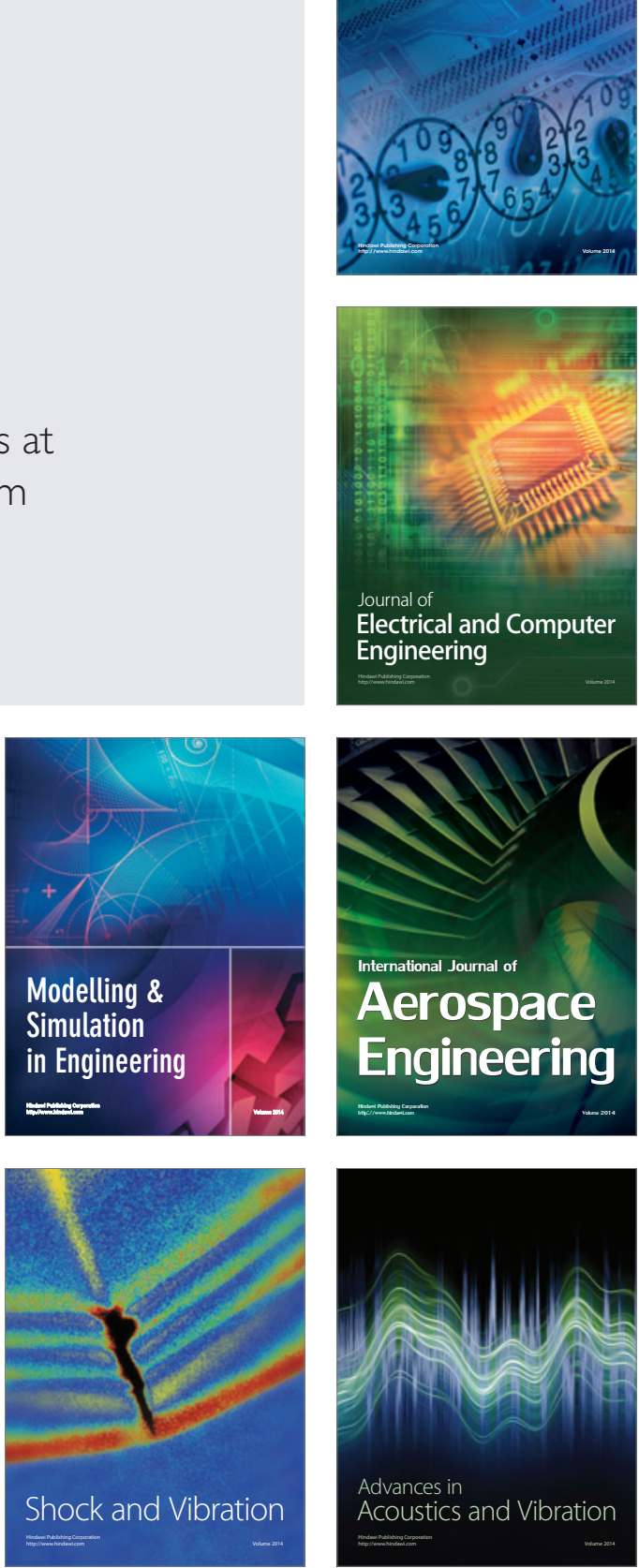